\title{
Artificial Taste Buds: Bioorthogonally Ligated Gustatory- Neuronal Multicellular Hybrids Enabling Intercellular Taste Signal Transmission
}

Trang Huyen Le-Kim, Bon Il Koo, Sung Duk Jo, Nai-Wen Liang, Moon Young Yang, In Cho, Jae Byum Chang, Tzu-Wei Wang, and Yoon Sung Nam*

Table S1. Estimation of maximum distance between gustatory cells and neurons by several calculation theories.

\begin{tabular}{|c|c|c|}
\hline Calculation Method & $\begin{array}{l}\text { Click chemistry-assisted } \\
\text { gap junction }\end{array}$ & $\begin{array}{l}\text { DNA-assisted } \\
\text { gap junction }\end{array}$ \\
\hline 1. Contour length & $13.6 \mathrm{~nm}$ & $24.1 \mathrm{~nm}$ \\
\hline 2. Kuhn length & $7.5 \mathrm{~nm}$ & $10.3 \mathrm{~nm}$ \\
\hline 3. Reference ${ }^{\dagger}$ & $8.6 \mathrm{~nm}$ & $12.2 \mathrm{~nm}$ \\
\hline
\end{tabular}

†Biophys. J. 95, 1590-1599 (2008) 


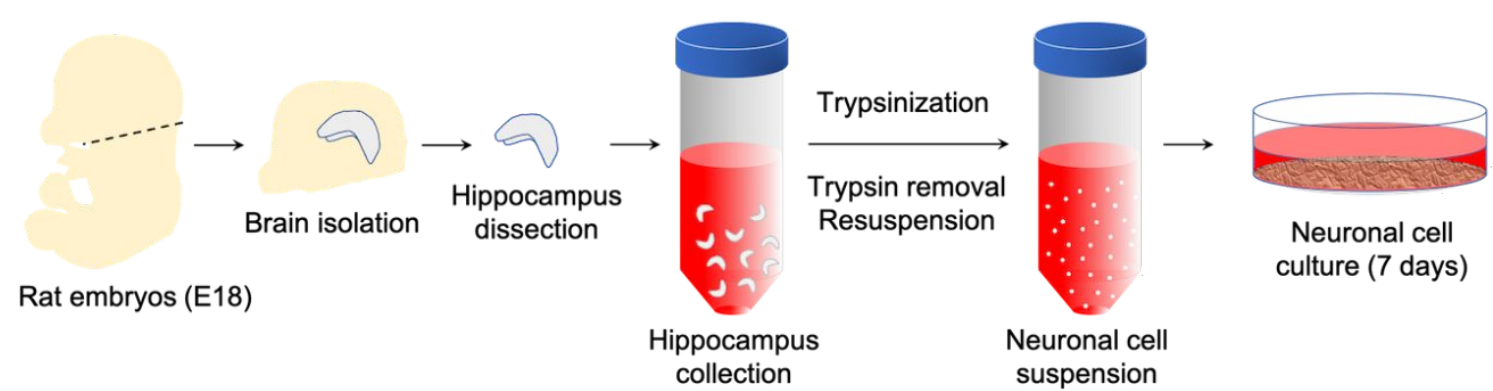

b

suspension
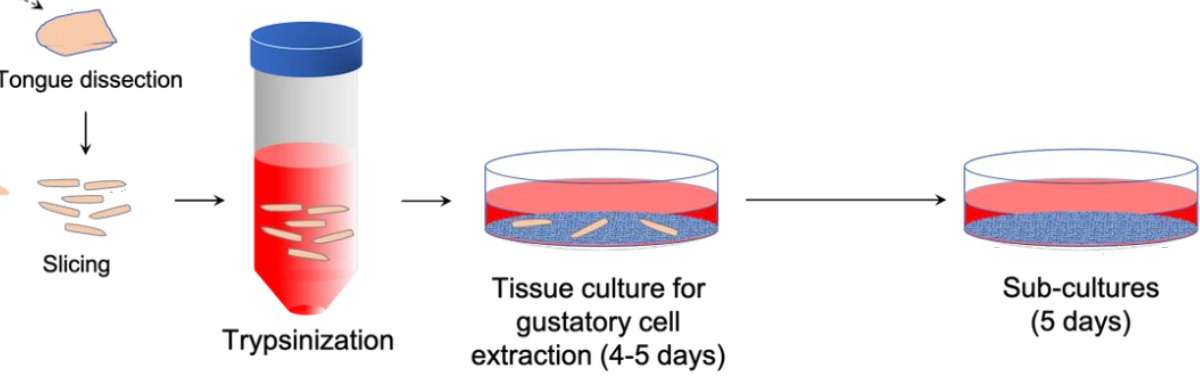

2-day-old neonatal mice

C

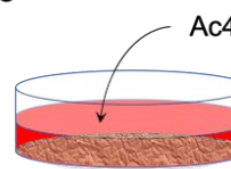

Ac4ManNAz

Neuronal cells
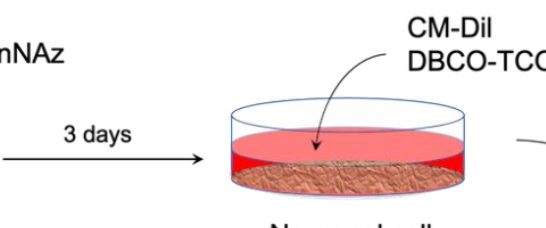

DBCO-TCO
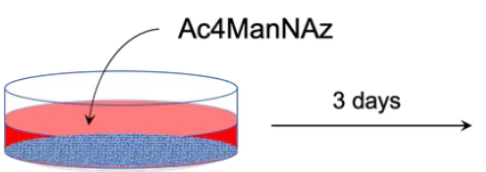

Neuronal cells

Gustatory cells
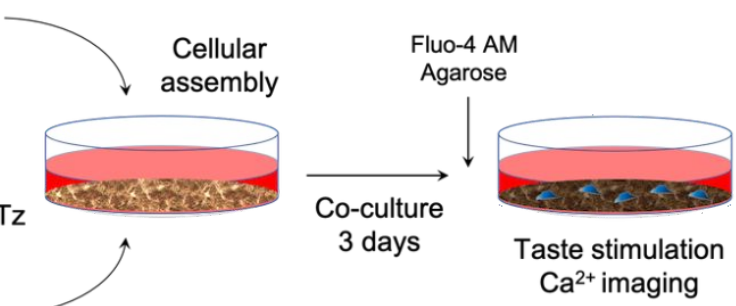

Figure S1. Schematic description of experiment procedures for the click chemistry-based assembly and co-culture of gustatory and neuronal cells 
a

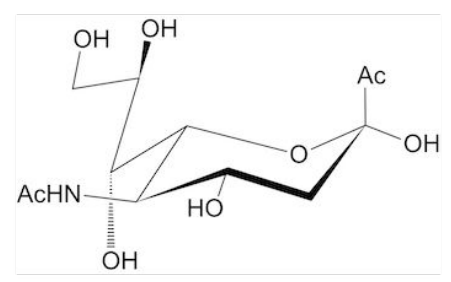

Sialic acid (SA) b

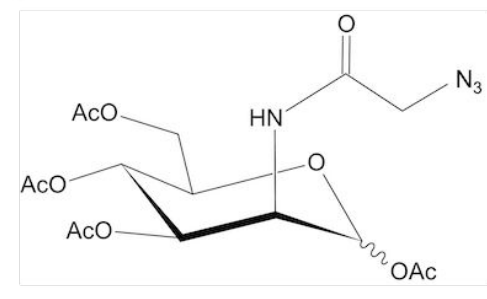

$\mathrm{N}$-azidoacetylmannosaminetetraacylated (Ac4ManNAz)

C

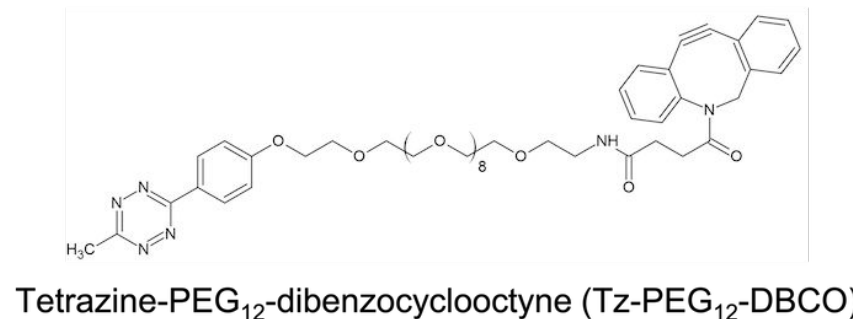

d

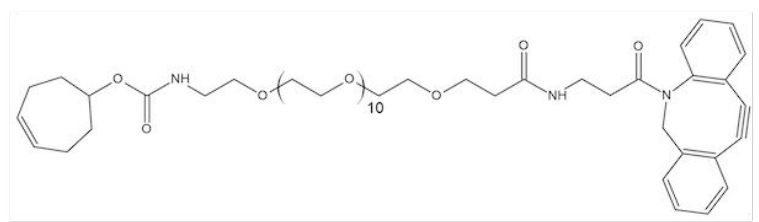

Trans-cyclooctene-PEG 12 -dibenzocyclooctyne (TCO-PEG 12 -DBCO)

Figure S2. Chemical formulas of sialic acid (SA), Ac4ManNAz, Tz-PEG 12 -DBCO, and TCO- $\mathrm{PEG}_{12}$-DBCO. 

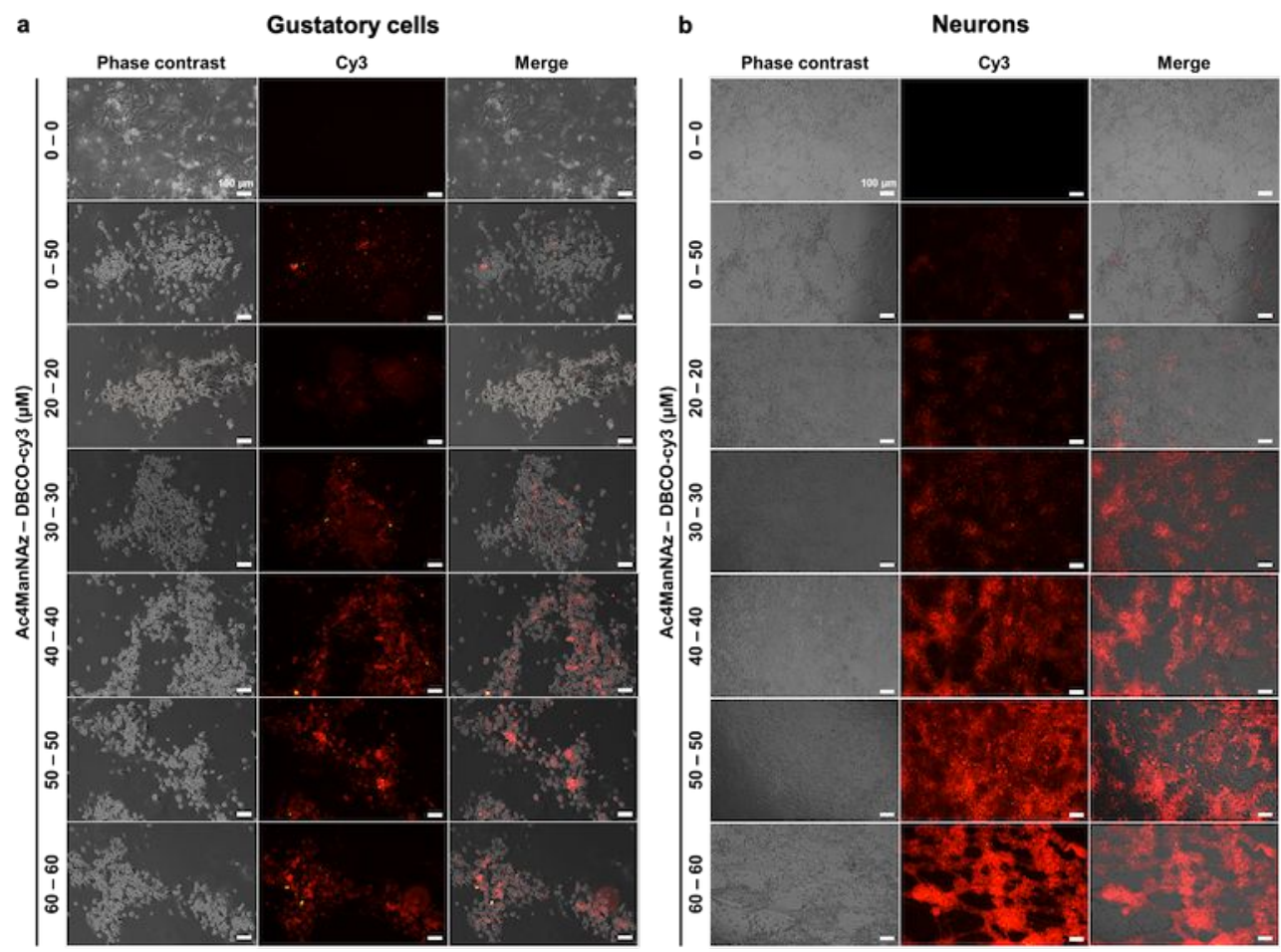

c
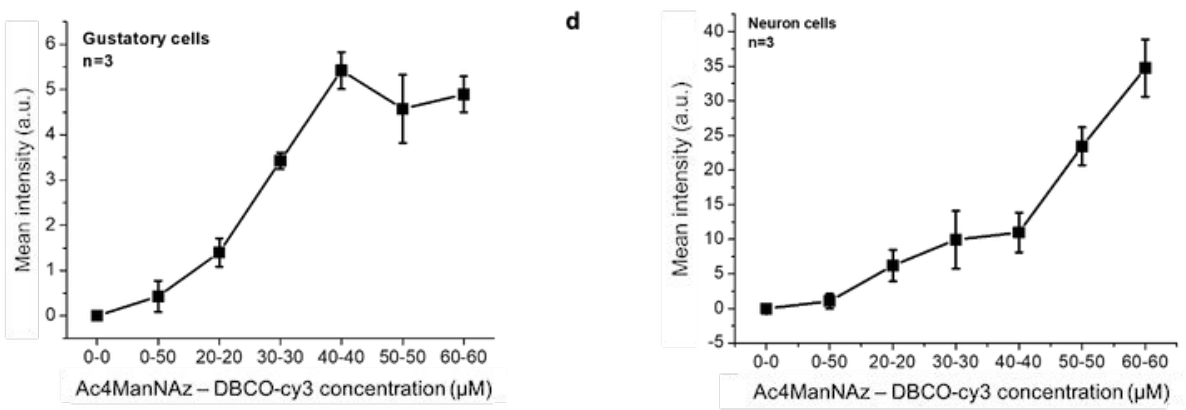

Figure S3. Investigation of sialic acid expression on the cell membrane of gustatory (A and C) and neuron (B and D) by adjusting the concentrations of Ac4ManNAz and DBCO-cy3. Scale bars: $100 \mu \mathrm{m}$. 
Gustatory cells 5 days after plating

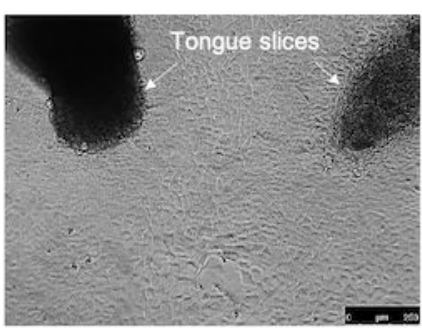

Neuron cells

7 days after plating

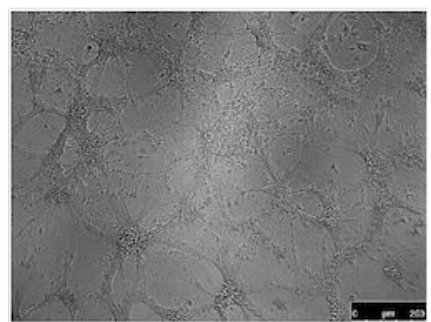

Figure S4. Optical microscopic images of gustatory and neuronal cells after 5 days and 7 days after plating, respectively.

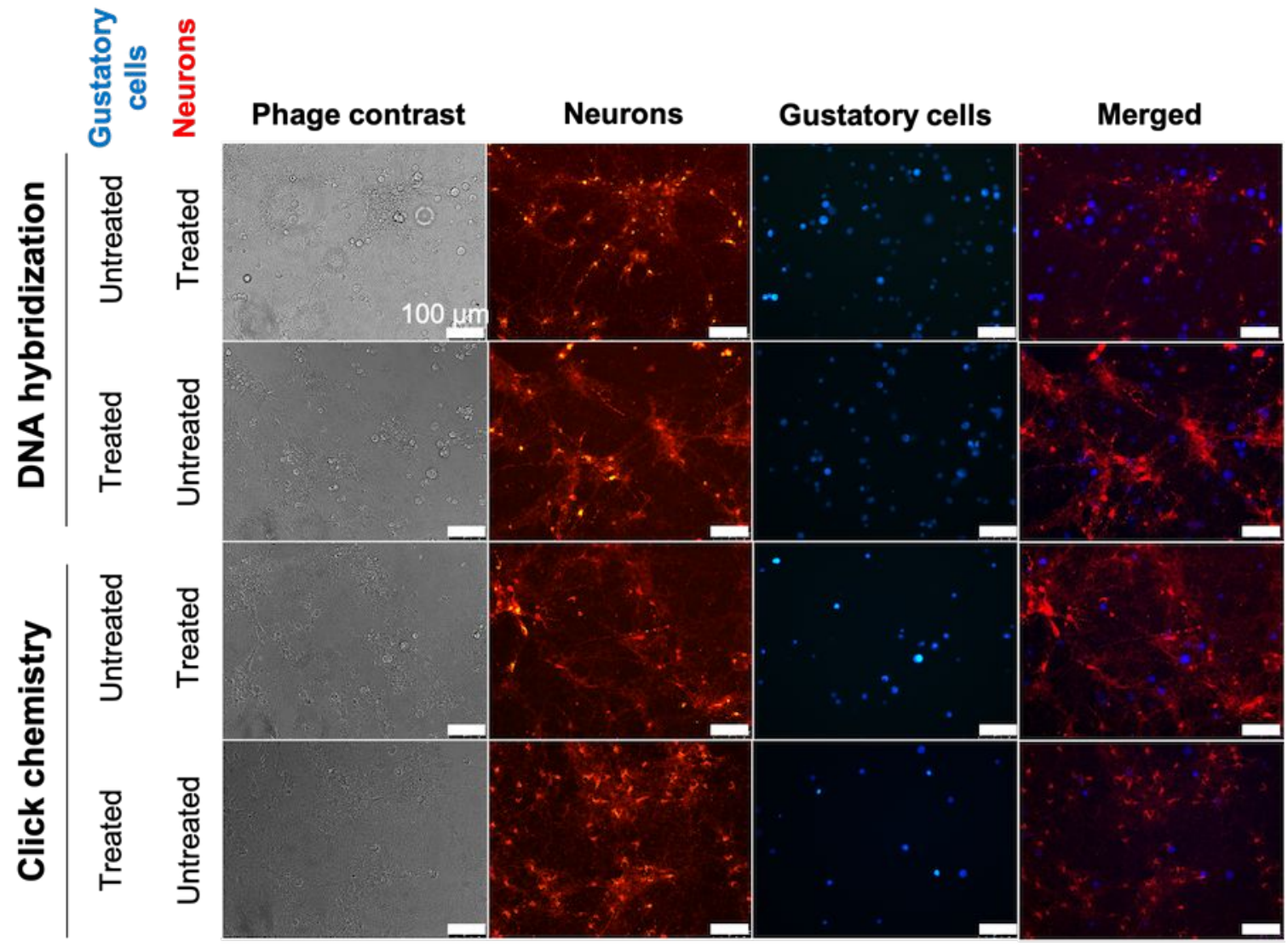

Figure S5. Investigation on the binding efficiency of gustatory cells on a neuronal network. Confocal microscopy images of neurons and gustatory cells, only one of which was treated by DNA- or click chemistry assembly procedures. 


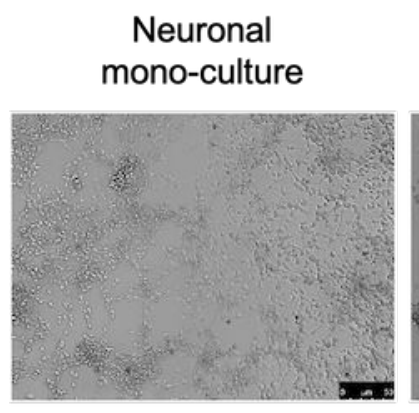

\section{Gustatory mono-culture}

Gustatory-neuronal hybrid co-culture
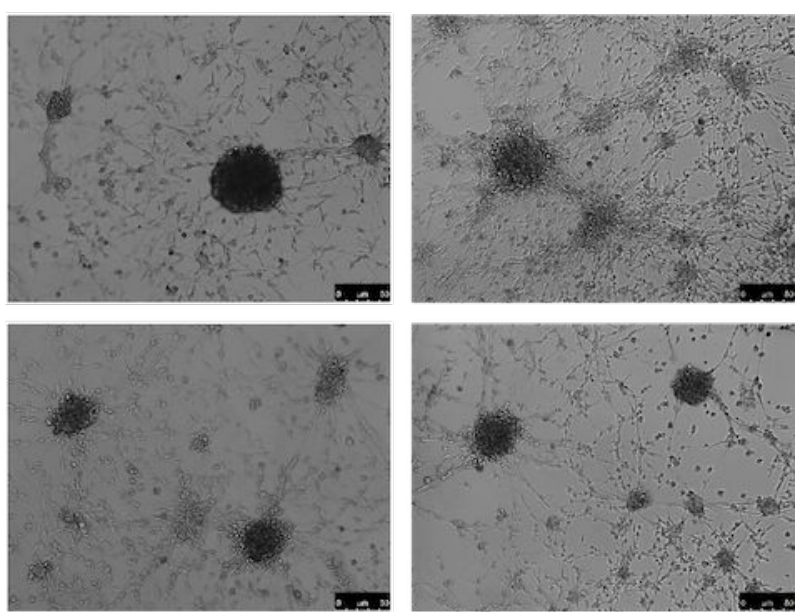

Figure S6. Optical microscopy images of gustatory-neuronal cell assemblies after co-culture for 3 days. 'NG click' has the most prominent clusters than neuron only $(\mathrm{N})$ and gustatory only (G). G has smaller clusters, while $\mathrm{N}$ only makes flat groups of cells rather than round clusters. 


\section{Supporting Movies}

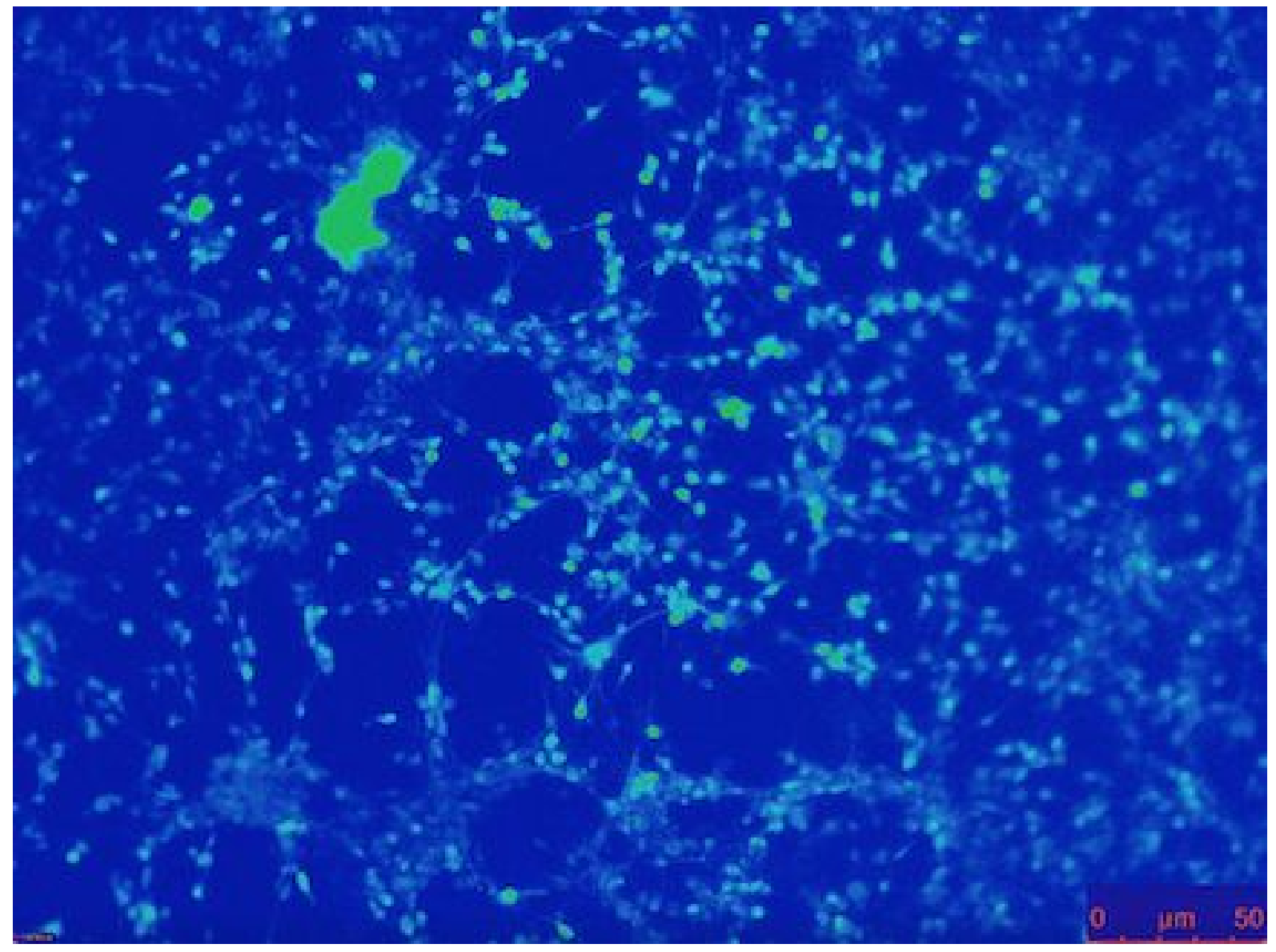

Movie S1. Mono-cultured neurons responding to $1 \mathrm{mM}$ denatonium benzoate. A movie file is provided: Movie 1 Neurons.mp4. 


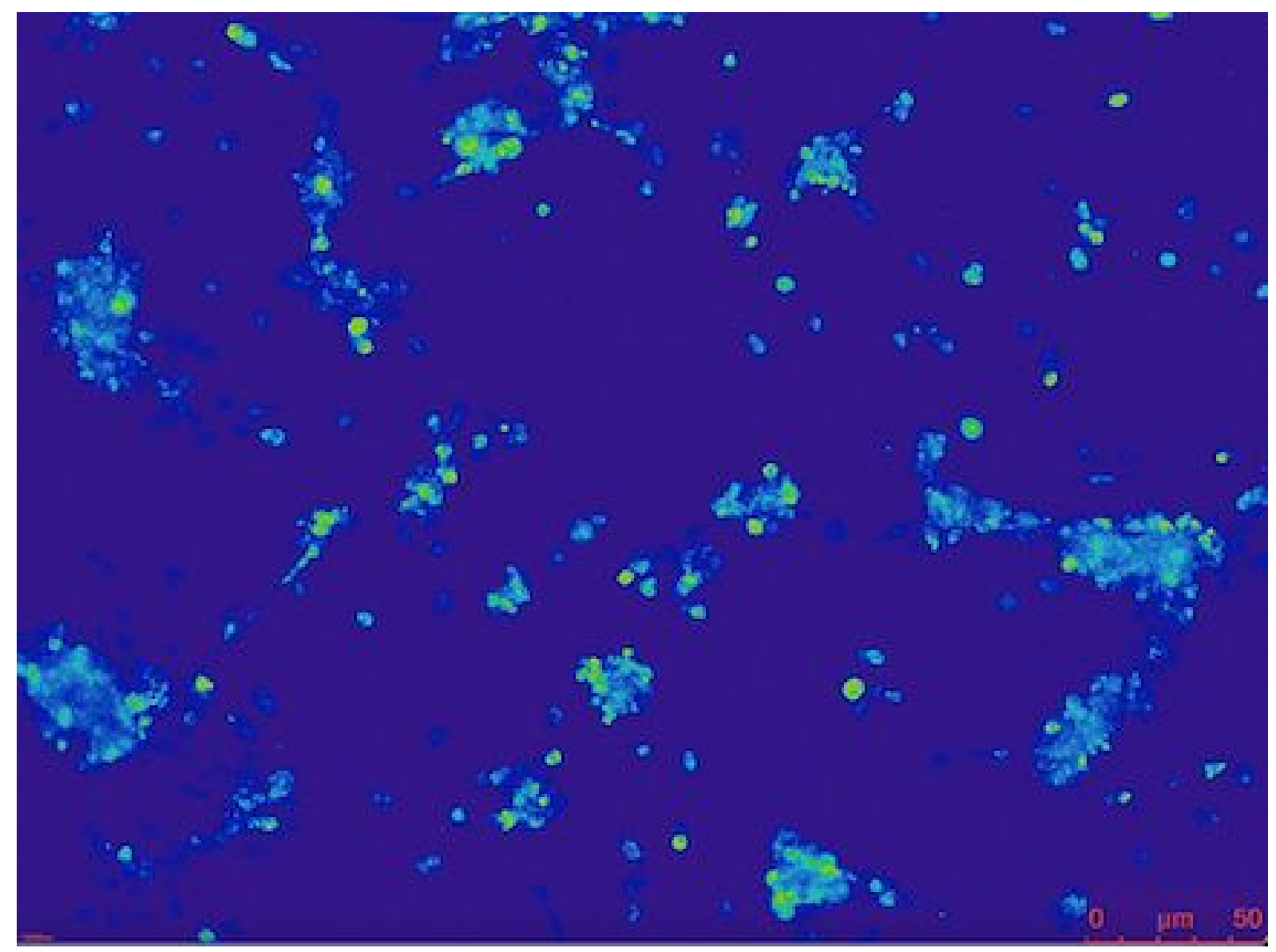

Movie S2. Mono-cultured gustatory cells responding to $1 \mathrm{mM}$ denatonium benzoate. A movie file is provided: Movie 2 Gustatory cells.mp4 


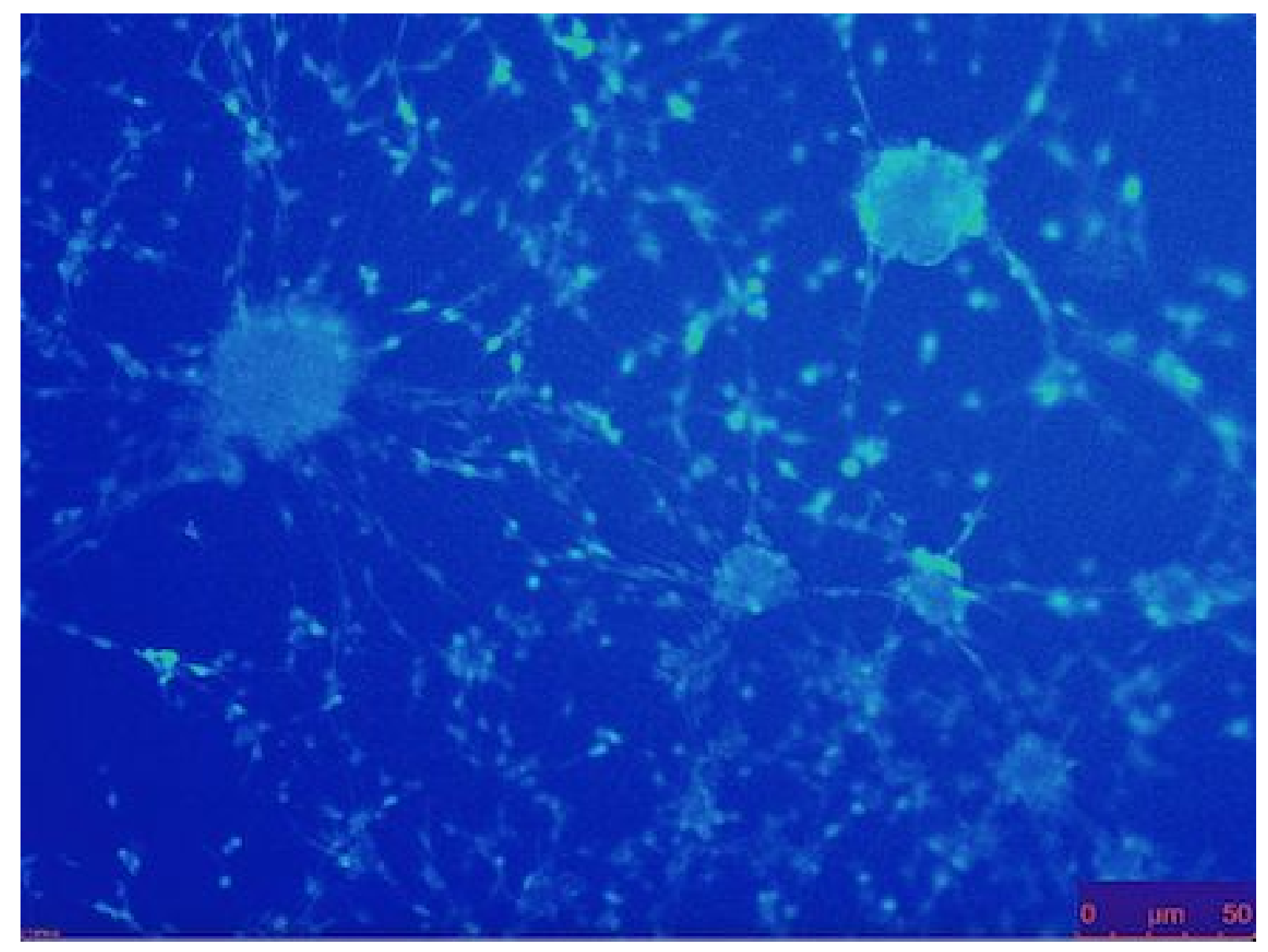

Movie S3. Neuron-gustatory cellular hybrids assembled by click chemistry responding to 1 $\mathrm{mM}$ denatonium benzoate. A movie file is provided: Movie 3 Hybrids.mp4. 